\title{
Influence of scattering and birefringence on the phase shift between electric field components of polarized light propagated through biological tissues
}

Mariia Borovkova, Alexander Bykov, Alexey Popov, Igor Meglinski

Mariia Borovkova, Alexander Bykov, Alexey Popov, Igor Meglinski, "Influence of scattering and birefringence on the phase shift between electric field components of polarized light propagated through biological tissues," Proc. SPIE 11075, Novel Biophotonics Techniques and Applications V, 110750R (22 July 2019); doi: 10.1117/12.2526394 


\title{
Influence of scattering and birefringence on the phase shift between electric field components of polarized light propagated through biological tissues
}

\author{
Mariia Borovkova*, Alexander Bykov, Alexey Popov, and Igor Meglinski \\ Optoelectronics and Measurement Techniques, University of Oulu, Oulu, 90570, Finland \\ *mariia.borovkova@oulu.fi
}

\begin{abstract}
The interest to the use of polarized light in various modern biomedical applications is significantly growing. We explore the influence of scattering and birefringence on the phase shift between electric field components of polarized light propagated through biotissues. Degree of polarization and phase shift between the orthogonal components of circularly polarized light, propagated through the tissue samples, are examined utilizing Poincaré sphere. Scattering reduction and birefringence increase are achieved, respectively, by optical clearing and mechanical stretch. Notably different signatures of state of polarization are observed for scattering and birefringence alterations that makes it possible to distinguish mechanisms of phase retardation.
\end{abstract}

Keywords: polarimetry, Stokes vector, Poincaré sphere, scattering, birefringence, skin tissue, optical clearing

\section{INTRODUCTION}

The use of polarized light in biomedical research has been growing explosively [1]. Recently a number of innovative polarization-based optical modalities have been developed for various biomedical applications in order to improve the accuracy of measurements and enhance the imaging contrast, with an ultimate aim to attain the practical tool for the optical biopsy and functional characterization of biological tissues. The Jones-vector basis, the Mueller-matrix decomposition approach, or the Stokes-vector formalism are used for the assessment of polarization changes within the biological tissues. It has been shown that the Mueller-matrix decomposition approach is highly promising for the characterization of various types of structural malformations from cancerous tissues to healthy ones [2]. However, this approach does not allow distinguishing the role of scattering and birefringence in the phase shift between the orthogonal components of polarized light.

In current study, utilizing Stokes vector and Poincaré sphere approach we attempt to distinguish the contributions of scattering and birefringence in the phase retardation of polarized light propagated within biotissue. It has been demonstrated that the use of the Poincaré sphere in polarimetry has high potential for the screening of cancer aggressiveness $[3,4]$. Here, we use the similar approach for the phenomenological study of phase retardation nature in biotissue based on the series of model experiments with tissue-mimicking phantoms demonstrating separately scattering and birefringence and the series of experiments on chicken skin samples induced with alterations in scattering and birefringence. Thus, in current report, we examine a set of biotissue-mimicking phantoms with different optical properties and chicken skin tissue with means of circularly polarized light sensing, Stokes vector modality and Poincaré sphere approach. Chicken skin is chosen as a model with a future goal to transfer the developed methodology in the studies of collagen state in cosmetology.

\section{METHODS AND MATERIALS}

The measurements were performed using the developed in-house experimental system utilizing circularly polarized light [4]. With the help of biotissue-mimicking phantoms, alterations of scattering and birefringence were imitated independently and their influence on the incident circularly polarized light was traced on the Poincare sphere. The experiments were performed in two regimes: 1) with equal angles of incidence and detection and no separation between the incidence and detection spots, 2) with the angles of incidence and detection of $55^{\circ}$ and $30^{\circ}$, respectively, and $1.5 \mathrm{~mm}$ separation between the spots of incidence and detection. Based on the model experiments, the samples of chicken skin

Novel Biophotonics Techniques and Applications V, edited by Arjen Amelink, Seemantini K. Nadkarni, Proc. of SPIE-OSA Vol. 11075, 110750R · C 2019 SPIE-OSA · CCC code: 1605-7422/19/\$21 · doi: 10.1117/12.2526394 
with alterations of scattering and birefringence were examined. The scattering in chicken skin was reduced by optical clearing (utilizing $40 \%$ glycerol-water solution), whereas birefringence was induced by mechanical stretch of the sample. A typical experiment consisted of three stages: drying, optical clearing and stretching. The spatial scanning of the sample was performed evenly during all three stages, without extra alignment of the sample. The inducement of birefringence via mechanical stretch in chicken skin was validated by second-harmonic generation imaging.

\section{RESULTS AND DISCUSSION}

\subsection{Model experiments}

The results of the experiments imitation scattering alteration are omitted here for brevity. According to the experimental data, the increase of scattering coefficient leads to the increase of the light depolarization by the scattering medium, while the Stokes vector remains roughly unchanged. The birefringence model experiments were performed using a mirror (to demonstrate pure induced birefringence with no scattering) and phantoms with $\mu_{\mathrm{s}}=4,8.1 \mathrm{~mm}^{-1}$ (to imitate induced birefringence with constant scattering). During the experiments, the phase retardation of the light was altered by the rotation of the half-wave plate which modeled birefringence alteration in the sample. The Stokes vectors of the light scattered from the samples with alterations of birefringence versus the induced phase retardation in degrees are presented in the Fig.1(a-d) degree of polarization, V, Q, U Stokes parameters, respectively. Fig.1-e shows the Stokes vectors mapped on the Poincaré sphere. Radii of the blue and yellow spheres correspond to $100 \%$ and $6 \%$ degree of polarization, respectively. Grey color shows results for the mirror, blue: $\mu_{\mathrm{s}}=3.9 \mathrm{~mm}^{-1}$, regime 1 ; red: $\mu_{\mathrm{s}}=8.1 \mathrm{~mm}^{-1}$, regime 1; green: $\mu_{\mathrm{s}}=3.9 \mathrm{~mm}^{-1}$, regime 2 ; orange: $\mu_{\mathrm{s}}=8.1 \mathrm{~mm}^{-1}$, regime 2 . The incident light is right circularly polarized which corresponds to the north pole on the Poincare sphere. As it is shown in Fig.1(a-d), the alterations of birefringence lead to the notable trend in the Stokes vector, whereas degree of polarization remains almost constant. The shapes of Stokes components trends are similar for all samples, however, some are scaled or/and have a phase shift relative to each other. The full 'cycle' of the Stokes vector corresponds to the change in phase retardation of the incident light from $90^{\circ}$ to $-90^{\circ}$ and back and is observed as a circle mapped on the surface of the Poincaré sphere with the constant radius corresponding to the unchanged degree of polarization (see Fig.1-e). Unlike scattering alteration, birefringence alteration does not cause the trend in the degree of polarization values. Although, according to the experimental results in the regime 2, birefringence alterations lead to some oscillation in the degree of polarization value which is most likely due to internal birefringence of the phantoms noticeable only with high source-detection separation.
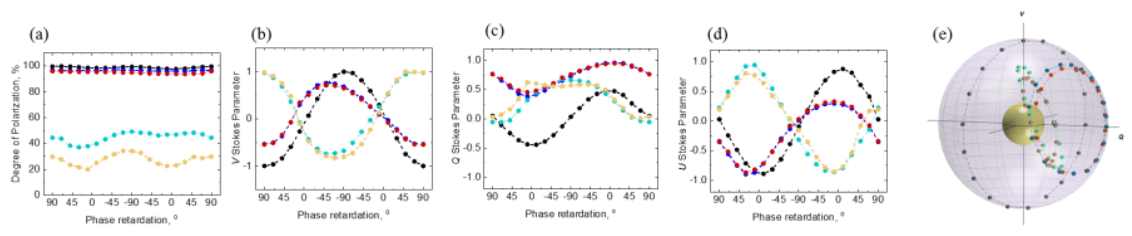

Figure 1. Results of model experiments with induced birefringence: degree of polarization (a), Stokes parameters V (b), Q (c), U (d). Stokes vectors mapped on the Poincaré sphere (e). Explanations are given in the text.

\subsection{Polarized light sensing of chicken skin}

Typical alterations of the state of polarization of the light propagated through the sample of chicken skin influenced by drying, optical clearing and mechanical stretch are presented in Fig.4-a and Fig.4-b alterations of the degree of polarization and V Stokes parameter in time, (c) measured Stokes vectors mapped on the Poincaré sphere, (d) enlarged view of Stokes vectors, (e) closely the data points that correspond to stretching. Black data points correspond to drying, green ones indicate the process of optical clearing, and red ones show stretching. Radii of the blue and yellow spheres correspond to $12 \%$ and $80 \%$ degree of polarization, respectively.

In the beginning of the experiment, the degree of polarization was approximately $40 \%$. Drying causes the reduction of scattering in the sample, which leads to the steady growth of degree of polarization. Once the optical clearing agent is applied topically to the tissue sample, the degree of polarization of the light undergoes a rapid fall due to the change of the sample's superficial content. During the optical clearing, degree of polarization grows exponentially until it saturates by approximately $90^{\text {th }}$ minute of the experiment. After that, mechanical stretch was applied to the sample in order to induce birefringence, which, according to results, does not affect the degree of polarization significantly. The changes of the V Stokes parameter are shown in Fig.2-b. In the beginning of the experiment, the state of polarization of light 
propagated through the sample is close to linear. The process of drying leads to the steady decay of V Stokes parameter. The moment of topical application of clearing agent is followed by the rapid change of the V Stokes component due to refractive index match. Then, the process of optical clearing causes exponential decay of V Stokes parameter up to $\sim 70$ minutes of experiment until saturation. The reduction of scattering caused by optical clearing and drying leads to the excess of the co-polarized component over cross-polarized component due to the increase of the forward scattering. The process of mechanical stretch alters birefringence of the sample, which is manifested in the renewed minor down-trend of V Stokes parameter. This is due to the fact that applied mechanical stretch influences the inner structure of the tissue sample, in particular, aligns initially dispersed collagen fibers in a major direction, which induces form birefringence. These results correlate well with the modelling experiments and the results of alternative studies [4,5].
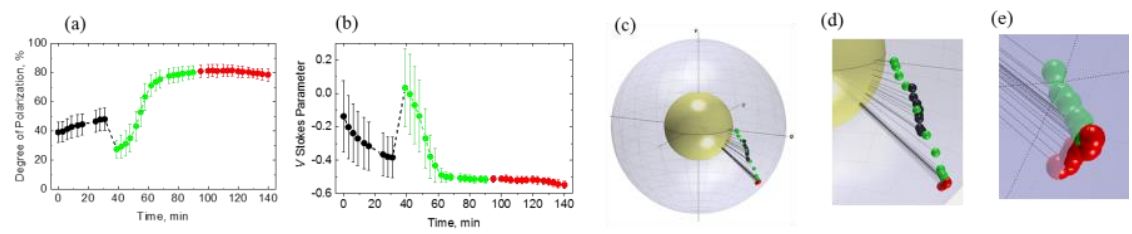

Figure 2. State of polarization of the light propagated within the chicken skin tissue influenced by drying, optical clearing and mechanical stretch: (a) degree of polarization, (b) V Stokes parameter, (c) Stokes vectors on the Poincaré sphere, (d) enlarged view of Stokes vectors mapped on the Poincaré sphere, e) enlarged view of data points that correspond to stretching. Explanations are given in the text.

According to the obtained results, the birefringence $\Delta \mathrm{n}(\Delta \mathrm{n}=\delta \lambda / 2 \pi \mathrm{l}$, where $\delta$ is the phase shift, $\lambda$ is the wavelength and 1 is the photons path-length within the tissue $1 \mathrm{~mm}$ ) for the chicken skin is estimated as $0.3 \times 10^{-3}$. The results well agree with the results obtained in the alternative studies [6].

\section{CONLUSION}

The study is focused on the separation of scattering and birefringence contributions in the phase retardation of the circularly polarized light transmitted through the biotissue. The results have shown different patterns of the state of polarization of light mapped on the Poincaré sphere for scattering and birefringence alterations, which makes it possible to identify these two mechanisms of phase retardation.

\section{ACKNOWLEDGEMENTS}

This project has received funding from the European Union's Horizon 2020 research and innovation programme under the Marie Skłodowska-Curie grant agreement No 713606 and the Academy of Finland (project: 314369).

\section{REFERENCES}

[1] T. Novikova, I. Meglinski, J. Ramella-Roman, and V. Tuchin, "Polarized light for biomedical applications," J. Biomed. Opt. 21, 071001 (2016).

[2] T. Novikova, A. Pierangelo, A. De Martino, A. Benali, and P. Validire, "Polarimetric imaging for cancer diagnosis and staging," Opt. Photonics News 23, 26-33 (2012).

[3] I. Meglinski, C. Macdonald, A. Doronin, and M. Eccles, "Screening cancer aggressiveness by using circularly polarized light," in Optics in the Life Sciences, OSA Technical Digest (online), Optical Society of America, p. BM2A.4 (2013).

[4] B. Kunnen, C. Macdonald, A. Doronin, S. Jacques, M. Eccles, and I. Meglinski, "Application of circularly polarized light for non-invasive diagnosis of cancerous tissues and turbid tissue-like scattering media," J. Biophoton. 8, 317-323 (2015).

[5] C. Macdonald and I. Meglinski, "Backscattering of circular polarized light from a disperse random medium influenced by optical clearing," Laser Phys. Lett. 8, 324 (2011).

[6] V.V. Tuchin, L. Wang and D.A. Zimnyakov, [Optical polarization in biomedical applications], Springer Science \& Business Media, (2006). 